\title{
A sensorless efficiency test system for a high-speed permanent magnet synchronous motor
}

\author{
Li JI ${ }^{1,2}$, Yixiang $\mathrm{SHAO}^{2}$, Sujie $\mathrm{GAO}^{3}$
}

MPCE

\begin{abstract}
We present a sensorless efficiency test system with energy recovery for a high-speed permanent magnet synchronous motor (PMSM). In the system, two identical high-speed PMSMs are used as the motor under test (MUT) and the load machine (LM), respectively. A new sensorless vector control (VC) method based on a hypothetical reference frame is presented to control both the MUT and the LM. Also, a regenerating unit is used to implement energy circulation to save energy. Experiments were carried out on a prototype, with a digital controller based on the TMS320F28335, to verify the adequacy of the sensorless VC method. As a result, the efficiency test system achieves the load test at the speed of $21000 \mathrm{r} / \mathrm{min}$ without any reduction equipment. During the test, the energy regenerated by the LM could be fed back to the MUT by the regenerating unit, and $81.31 \%$ electrical power was saved. In addition, with the proposed sensorless VC method, both
\end{abstract}

CrossCheck date: 12 September 2018

Received: 18 September 2017/Accepted: 12 September 2018/ Published online: 22 December 2018

(c) The Author(s) 2018

$凶$ Li JI

jili198504@hhu.edu.cn

Yixiang SHAO

shaoyixiang@sgepri.sgcc.com.cn

Sujie GAO

sujie-gao@sgxy.sgcc.com.cn

1 College of Energy and Electrical Engineering, Hohai University, Nanjing 210098, China

2 NARI Group Corporation (State Grid Electric Power Research Institute), Nanjing 211102, China

3 State Grid Xin Yuan Company Limited, Beijing 100000, China the MUT and the LM can work at $i_{d}=0$ without a position sensor.

Keywords Distributed generation, High-speed permanent magnet synchronous motor (PMSM), Sensorless control, Energy saving

\section{Introduction}

With the advantages of small size, high efficiency, and high reliability, permanent magnet synchronous motors (PMSMs), especially high-speed PMSMs, have been used extensively for distributed generation and industrial drives in recent years [1-4]. In order to determine the performance and characteristics of PMSMs, a loading facility is required for the load test. However, a conventional loading facility, such as an eddy dynamometer, mechanical dynamometer and hysteretic dynamometer, requires a reduction gearbox for the high-speed PMSM. Some types of facility also require a water cooling system for high power operation. Not only will it be extremely noisy but it also wastes energy and water during the test [5-7].

In order to save energy, there have been attempts to produce electric dynamometers that can implement energy circulation. In [8], Wang et al designed an electric dynamometer with a DC generator as the load motor. The recovered energy is fed back to the power grid through the energy regenerating unit. With the energy recovery system, this DC dynamometer is more efficient than traditional ones. However, according to the commutator, the DC dynamometer cannot run at a high speed. Therefore, recent research into electric dynamometers focuses on squirrel cage induction motors which can be operated at a higher speed. 
Yang et al presented a novel AC electric dynamometer based on a squirrel cage induction motor with an improved direct torque control (DTC). The experiments showed that the proposed dynamometer realized energy recovery and had good dynamic performance during the test $[9,10]$. Liu et al presented an asynchronous dynamometer using an induction machine by DTC [11]. In [11], the asynchronous dynamometer was in the generating mode by DTC, while the experimental motor supplied the driving torque by employing field-oriented control, and the two electric machines were connected with a common-DC-bus to implement energy circulation. As the energy was circulating between motor and dynamometer, this method could save test energy without any harmonic pollution to the power grid. However, DTC brought torque ripple which could be a problem for the performance test. Therefore, some scholars like Zhang paid attention to the vector control (VC) [12]. In [12], the proposed dynamometer was connected to the power grid by a back-to-back pulse width modulation (PWM) converter to feed back energy. An improved VC based on flux-oriented rotor was adopted to make the induction motor operate in generating mode with controllable torque. The effectiveness of the proposed VC was verified by MATLAB-based simulation. In [13], Chu presented a dynamic testing system with energy recovery for an electric vehicle. By the VC, the dynamic testing system can be controlled from light load to full load to accomplish the dynamic test. His test showed that the testing system saved $65 \% \sim 70 \%$ of the energy by simulating the full-range speed and torque output.

However, existing asynchronous dynamometers are still restricted for high-speed PMSM. First, due to the extreme high speed, the PMSM and dynamometer need to have accurate alignment. However, it is difficult to achieve precise coaxial operation with two facilities of different size. In addition, the $\mathrm{VC}$ requires the rotor position which is detected by position sensor such as encoders and resolvers to commutate and control current. However, these features increase the cost and size of the dynamometer and reduce reliability.

This paper presents a sensorless efficiency test system with energy recovery for the high-speed PMSM. A novel sensorless VC method based on a hypothetical reference frame is presented to control both the motor under test (MUT) and the load machine (LM). Then experiments are carried out with a $75 \mathrm{~kW}$ high-speed PMSM to verify the adequacy of the method. As a result, the proposed system can be used at different speeds and power levels with energy recovery. During the whole test, considering the $d$ $q$ frame, both MUT and LM can be operated with zero phase current in the $d$ axis $\left(i_{d}=0\right)$ without any speed or position sensor.

\section{Structure of efficiency system}

The scheme of the sensorless efficiency test system is shown in Fig. 1. Given the considerable power and speed level of the PMSM, an identical high-speed PMSM is used as the LM in the system. Two PMSMs are mechanically connected by a high-speed torque meter. The MUT is driven as a motor by the sensorless VC method, with speed close-loop. The LM also uses a sensorless VC method but with a torque close-loop as a generator to supply the load torque. The MUT and LM are driven by two independent inverters, inverter 1 and inverter 2. Both inverters are electrically connected to a common DC bus which is connected to the power grid through a rectifier. As the unilateral conduction of the rectifier, the energy generated by the LM will not be fed back to the grid but circulate to the MUT. During the test, a power analyzer is used to measure the input electrical power of the whole system (marked (1) in Fig. 1) and both MUT (2) and LM (3) synchronously, and a PC is used to record the data from the torque meter to calculate mechanical power.

\section{Sensorless VC method for MUT}

For the MUT, the purpose is to make it work as a motor to supply the driving torque. In addition, we hope the whole system has good dynamic performance and high efficiency. $\mathrm{VC}$ is considered to be an ideal method for PMSMs. However, traditional VC methods require rotor flux position, which cannot be measured in high-speed PMSMs. Therefore, the sensorless method is essential for the system. The typical sensorless VC method for PMSMs is to estimate the rotor flux position using the instantaneous voltage equation of the motor and the detected current, and observers are usually used to enhance the robustness of the control system [14-19]. However, similarly to the use of observers, these sensorless methods require excessive

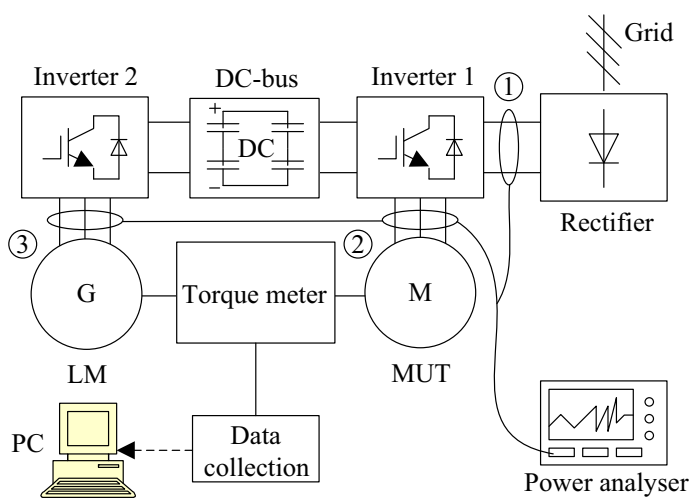

Fig. 1 Scheme of sensorless efficiency test system 
computing time in the case of high-speed PMSMs, because of the higher carrier frequency, which is unacceptable. Therefore, a convenient sensorless VC method striving for $i_{d}=0$ is presented in this paper.

First, the voltage equation of a PMSM, based on the $d-q$ frame, can be described as follows [20]:

$\left[\begin{array}{l}u_{d} \\ u_{q}\end{array}\right]=\left[\begin{array}{cc}R_{\mathrm{S}}+L_{d} \mathrm{p} & -\omega_{\mathrm{r}} L_{q} \\ \omega_{\mathrm{r}} L_{d} & R_{\mathrm{S}}+L_{q} \mathrm{p}\end{array}\right]\left[\begin{array}{c}i_{d} \\ i_{q}\end{array}\right]+\left[\begin{array}{c}0 \\ e_{0}\end{array}\right]$

where $u_{d}, u_{q}, i_{d}, i_{q}, L_{d}, L_{q}$ are the components of phase voltage, phase current and inductance respectively in the $d$ axis and the $q$ axis; $R_{\mathrm{S}}$ is the resistance of the coil in each phase; $\omega_{\mathrm{r}}$ is the electric angular speed; and $e_{0}=\omega_{\mathrm{r}} \psi_{\mathrm{f}}$ denotes electromotive force (EMF) where $\psi_{\mathrm{f}}$ is the EMF constant.

However, without a position sensor, the controller has no information about the rotor position, which means it is impossible to decouple the voltage and current by projecting into the $d-q$ frame. Therefore, the controller determines the applied voltage according to a hypothetical $\delta-\gamma$ reference frame, which is not necessarily coincident with the actual rotor position. Figure 2 shows the vector diagram of the MUT, where $\omega_{\mathrm{S}}$ are the electric angular speed for the $\delta$ - $\gamma$ frame; $\theta_{\mathrm{r}}$ is the angle between the $d$ axis and the $A$ phase axis; and $\theta_{\mathrm{S}}$ is the angle between the $\delta$ axis and the $A$ phase axis. The angular error between the two coordinate systems can be expressed as $\Delta \theta=\pi / 2-\left(\theta_{\mathrm{S}}-\theta_{\mathrm{r}}\right)$. In this figure, two rectangular coordinate systems (the $d-q$ frame and the $\delta$ - $\gamma$ frame) are defined.

As is apparent in the figure, the $d$ axis is the actual rotor flux axis, whereas the $\delta$ - $\gamma$ frame is the hypothetical coordinate system assumed in the controller.

From Fig. 2, the coordinate transformation matrix from the $d-q$ frame to the $\delta$ - $\gamma$ frame is:

$\boldsymbol{C}_{\text {hyp }}=\left[\begin{array}{cc}\sin \Delta \theta & \cos \Delta \theta \\ -\cos \Delta \theta & \sin \Delta \theta\end{array}\right]$

Substitute (2) into (1), and the voltage equation for the MUT based on the $\delta-\gamma$ frame can be expressed as:

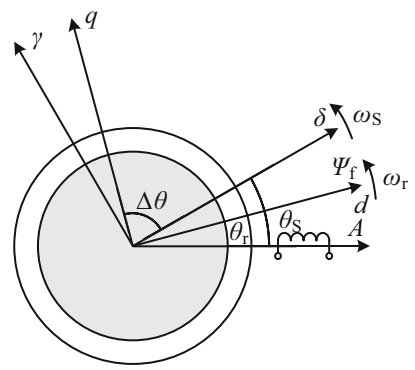

Fig. 2 Vector diagram of a MUT in $d-q$ frame and $\delta-\gamma$ frame

$$
\begin{aligned}
{\left[\begin{array}{l}
u_{\delta} \\
u_{\gamma}
\end{array}\right]=} & {\left[\begin{array}{cc}
R_{\mathrm{S}}+L_{d} \mathrm{p} & -\omega_{\mathrm{r}} L_{q}+L_{d} \frac{\mathrm{d} \Delta \theta}{\mathrm{d} t} \\
\omega_{\mathrm{r}} L_{q}-L_{d} \frac{\mathrm{d} \Delta \theta}{\mathrm{d} t} & R_{\mathrm{S}}+L_{d} \mathrm{p}
\end{array}\right]\left[\begin{array}{l}
i_{\delta} \\
i_{\gamma}
\end{array}\right] } \\
& +e_{0 \mathrm{x}}\left[\begin{array}{c}
\cos \Delta \theta \\
\sin \Delta \theta
\end{array}\right]
\end{aligned}
$$

where $u_{\delta}, u_{\gamma}, i_{\delta}, i_{\gamma}$ are the voltage and current respectively in the $\delta$ axis and the $\gamma$ axis; and $e_{0 \mathrm{x}}$ is the extended EMF, which is $e_{0 \mathrm{x}}=e_{0}-\mathrm{p}\left(L_{d}-L_{q}\right) i_{q}+\omega_{\mathrm{r}}\left(L_{d}-L_{q}\right) i_{d}$.

Through an equivalent transformation, the actual angular speed $\omega_{\mathrm{r}}$ is given by:

$\omega_{\mathrm{r}}=\omega_{\mathrm{S}}-\frac{\mathrm{d}\left(\frac{\pi}{2}-\Delta \theta\right)}{\mathrm{d} t}=\omega_{\mathrm{S}}+\frac{\mathrm{d} \Delta \theta}{\mathrm{d} t}$

where $\omega_{\mathrm{S}}$ is the hypothetical angle speed that can be modified in the controller.

Substitute (4) into (3), and take into account the nonsalient of the proposed high-speed PMSM $\left(L_{d}=L_{q}\right)$, the voltage equation of the MUT in the $\delta-\gamma$ frame can be rewritten as:

$$
\left[\begin{array}{l}
u_{\delta} \\
u_{\gamma}
\end{array}\right]=\left[\begin{array}{cc}
R_{\mathrm{S}}+L_{\mathrm{S}} \mathrm{p} & -\omega_{\mathrm{S}} L_{\mathrm{S}} \\
\omega_{\mathrm{S}} L_{\mathrm{S}} & R_{\mathrm{S}}+L_{\mathrm{S}} \mathrm{p}
\end{array}\right]\left[\begin{array}{c}
i_{\delta} \\
i_{\gamma}
\end{array}\right]+e_{0}\left[\begin{array}{c}
\cos \Delta \theta \\
\sin \Delta \theta
\end{array}\right]
$$

where $L_{\mathrm{S}}$ denotes the armature inductance, $L_{\mathrm{S}}=L_{d}=L_{q}$.

In addition, the torque equation in the $\delta-\gamma$ frame is shown as (6), under the ideal condition that the $\delta$ axis is coincident with the $q$ axis $(\Delta \theta=0)$, while $i_{\gamma}=0$, and $i_{\delta}$ contributes to the electromagnetic torque $T_{\mathrm{e}}$. In that case, the MUT works in the state of $i_{d}=0$. According to the angular error $\Delta \theta$, self-synchronization can be obtained by regulating the actual angular speed $\omega_{\mathrm{r}}$ as decreasing $\omega_{\mathrm{r}}$ for the condition of $\Delta \theta>0$ and increasing it for $\Delta \theta<0$. However, $\Delta \theta$ also cannot be measured without a position sensor. So an actual current $i_{\gamma}$ is employed to substitute for the unknown angular error $\Delta \theta$ in the proposed sensorless VC method.

$T_{\mathrm{e}}=p_{\mathrm{n}}\left[\psi_{\mathrm{f}}\left(i_{\delta} \cos \Delta \theta+i_{\gamma} \sin \Delta \theta\right)\right]$

where $p_{\mathrm{n}}$ is the number of pole pair.

First, initialize the applied voltage of $u_{\gamma}$ to $u_{\gamma}=\omega_{\mathrm{S}} L_{\mathrm{S}} i_{\delta}$ through inverter 1 , and then the voltage equation in the $\gamma$ axis can be rewritten as:

$-e_{0} \sin \Delta \theta=R_{\mathrm{S}} i_{\gamma}+L_{\mathrm{S}} \frac{\mathrm{d} i_{\gamma}}{\mathrm{d} t}$

From (7), we can know that in the case of $u_{\gamma}=\omega_{\mathrm{S}} L_{\mathrm{S}} i_{\delta}, i_{\gamma}$ is determined by the EMF $e_{0}$, the angular error $\Delta \theta$ and parameters of the MUT such as the resistance $R_{\mathrm{S}}$ and the inductance $L_{\mathrm{S}}$ where $R_{\mathrm{S}}$ and $L_{\mathrm{S}}$ are constants. Since the $\omega_{\mathrm{r}}$ doesn't change significantly in a short time, $e_{0}$ can also be regarded as a constant in every sampling period. Therefore, the variety of $i_{\gamma}$ is only related to the various $\Delta \theta$, and under 
the restriction of $\omega_{\mathrm{S}} \neq 0$ and $\Delta \theta \approx 0$, and $i_{\gamma}$ is proportional to the angular error $\Delta \theta$ approximately as:

$\Delta i_{\gamma}=0-i_{\gamma} \propto \Delta \theta$

Thus, self-synchronization can be achieved according to $i_{\gamma}$ as decreasing $\omega_{\mathrm{r}}$ for $i_{\gamma}<0(\Delta \theta>0)$ and increasing $\omega_{\mathrm{r}}$ for $i_{\gamma}>0(\Delta \theta<0)$. As there is no unknown quantity needing to be estimated, this sensorless VC method is simpler and more convenient than the traditional sensorless methods.

The mathematical model of the control system is now presented as follows.

Firstly, according to (7) and (8), the transfer function from $\Delta \theta$ to $i_{\gamma}$ with the restriction of $\Delta \theta \approx 0$ can be obtained as:

$G_{1}(s)=\frac{i_{\gamma}(s)}{\Delta \theta(s)}=\frac{-e_{0}}{R_{\mathrm{S}}+L_{\mathrm{S}} s}$

Secondly, from torque equation (6), the transfer function of the motion part around the ideal state $(\Delta \theta \approx 0)$ is obtained as:

$G_{2}(s)=\frac{\omega_{\mathrm{r}}(s)}{T_{\mathrm{e}}(s)-T_{1}(s)}=\frac{1}{\frac{J}{p_{\mathrm{n}}} s+\frac{R_{\Omega}}{p_{\mathrm{n}}}}$

where $J$ is the rotor inertia, and $R_{\Omega}$ is friction.

From the equations above, the mathematical model of the MUT can be expressed as single input single output (SISO) system as shown in Fig. 3, that is to say, a classical proportional-integral (PI) controller can be used to realize the sensorless VC method by regulating $i_{\gamma}$ to zero.

According to the parameters in Table 2, the frequency characteristic curve of the sensorless control system can be obtained as shown in Fig. 4. From Fig. 4a, the magnitude margin $h$ of the open-loop system is $-41.2 \mathrm{~dB}$, while the phase margin $\gamma=-109^{\circ}$, and the unity feedback system of the sensorless control system is not stable. As in the utilization of the PI controller, the frequency characteristics of the sensorless control system are corrected to $h^{\prime}=15.3 \mathrm{~dB}$, $\gamma^{\prime}=43.9^{\circ}$ as shown in Fig. 4 b, and it is shown from Fig. $4 d$ that after the PI correction the system can meet stability and fast response requirements.

Thus, the sensorless VC control system configuration is summed up as shown in Fig. 5, and the control process for the sensorless VC method will be illustrated in the following section.

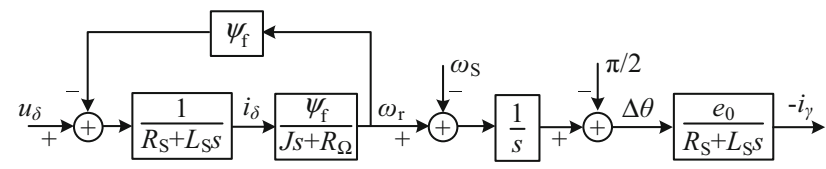

Fig. 3 Mathematical model of the MUT

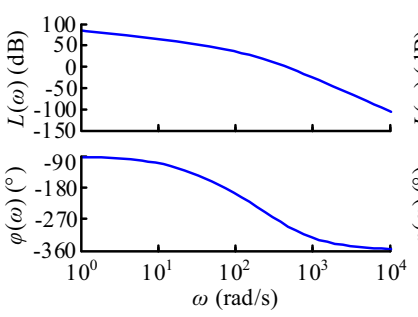

(a) Open-loop frequency characteristic curve of the MUT system

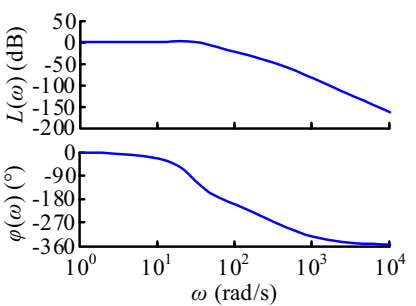

(c) Close-loop frequency characteristic curve after correcting

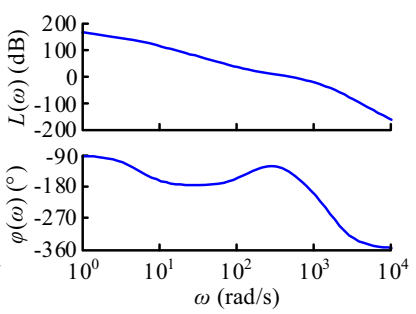

(b) Open-loop frequency characteristic curve after correcting

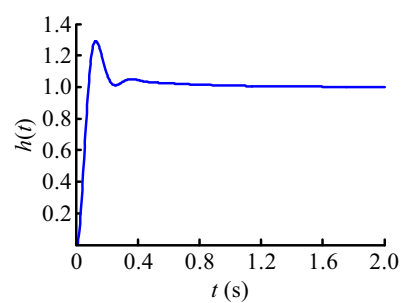

(d) Unit step response of closed-loop control system
Fig. 4 Bode diagrams for the sensorless control system

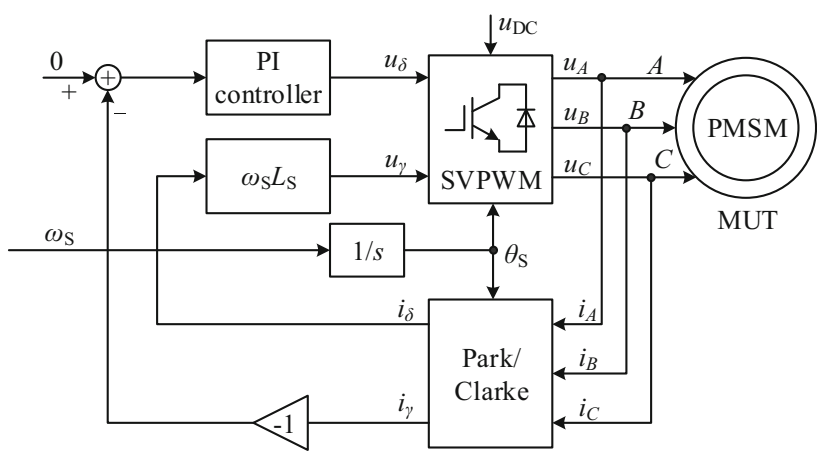

Fig. 5 Configuration of sensorless VC system based on hypothetical reference frame

\section{Sensorless VC method for LM}

In the proposed system, the LM works as a generator to load the MUT. Thus the purpose of the control method is to regulate torque current to run the LM in the fourth quadrant. In addition, the LM is expected to have good dynamic performance and high efficiency.

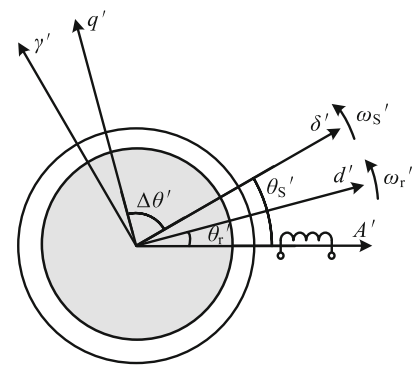

Fig. 6 Vector diagram of LM 
The method is also based on a hypothetical reference frame. Figure 6 shows the vector diagram of the LM.

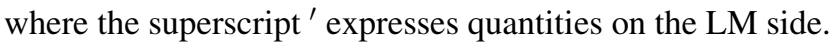
As stated above, the LM is mechanically coupled to the MUT. As a result, ignoring the negligible deformation of the rotor, the actual angular speed of the LM is synchronous with the MUT, as $\omega_{\mathrm{r}}=\omega_{\mathrm{r}}^{\prime}$. The hypothetical angular speed of the $\delta^{\prime}-\gamma^{\prime}$ frame can be set to be the same as the $\delta-\gamma$ frame, as $\omega_{\mathrm{S}}=\omega_{\mathrm{S}}{ }^{\prime}$. Therefore, according to equation (6), the error between $\triangle \theta$ and $\triangle \theta^{\prime}$ is invariable all the time, as $\theta_{\mathrm{E}}=\triangle \theta-\triangle \theta^{\prime}$, where $\theta_{\mathrm{E}}$ is a constant and only determined by the initial difference of the rotor position between the MUT and the LM. The theory of the control method will be discussed under two different conditions, $\theta_{\mathrm{E}}=0$ and $\theta_{\mathrm{E}} \neq 0$.

Firstly, assume $\theta_{\mathrm{E}}=0$, the initial rotor position of the LM is the same as the MUT, which means the angular error is the same for the MUT and the LM side, as $\triangle \theta=\triangle \theta^{\prime}$. As stated in Section 3, using sensorless VC, the $\delta$ axis is coincident with the $q$ axis $(\triangle \theta=0)$ in the steady state on the MUT side.

Therefore, in the LM side, the $\delta^{\prime}$ axis will also be coincident with the $q^{\prime}$ axis $\left(\triangle \theta^{\prime}=0\right)$ with the effect of the MUT. In that case, we use two current regulators to keep $i_{\delta}{ }^{\prime}<0$ and $i_{\gamma}{ }^{\prime}=0$. Then according to the torque equation (6), the LM will supply negative braking torque to the MUT. The more negative $i_{\delta}{ }^{\prime}$ is, the more load is on the MUT, and more energy is fed back. In addition, because $i_{\gamma}{ }^{\prime}=0$, the LM will work in the condition of $i_{d}{ }^{\prime}=0$ with minimum copper loss.

Figure 7 shows the vector diagram of the LM in steady state with $\theta_{\mathrm{E}}=0$, and $u_{\delta}{ }^{\prime}=R_{\mathrm{S}} i_{\delta}{ }^{\prime}+e_{0}{ }^{\prime}$ and $u_{\gamma}{ }^{\prime}=\omega_{\mathrm{S}}{ }^{\prime} L i_{\delta}{ }^{\prime}$. Thus the resultant vector $u_{\mathrm{S}}{ }^{\prime}$ always lags behind the EMF $\boldsymbol{e}_{0}{ }^{\prime}$, the LM is in the stage of regenerating and the electric energy will be fed back to the MUT through the common DC bus.

It is in fact difficult to achieve the condition of $\theta_{\mathrm{E}}=0$ without correction. Therefore, the feasibility of the above control method under the state of $\theta_{\mathrm{E}} \neq 0$ will be discussed. First, the same as with $\theta_{\mathrm{E}}=0$, a current regulator is used to keep $i_{\gamma}{ }^{\prime}=0$. The vector diagram of LM with $\theta_{\mathrm{E}} \neq 0$ is shown as Fig. 8. There will be an angular error between the

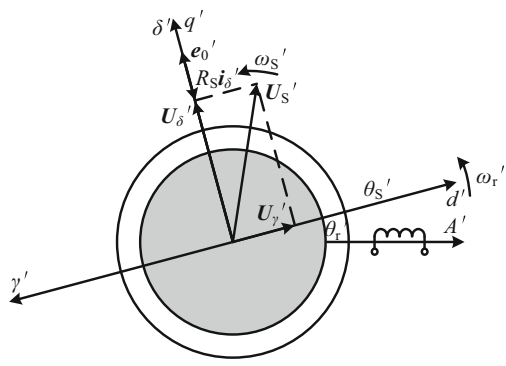

Fig. 7 Vector diagram of LM in steady state with $\theta_{\mathrm{E}}=0$

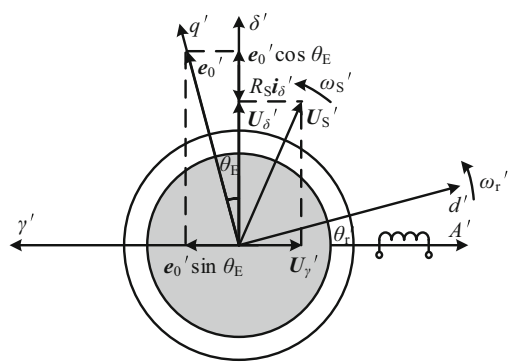

Fig. 8 Vector diagram of LM in steady state with $\theta_{\mathrm{E}} \neq 0$

$\delta^{\prime}$ axis and the $q^{\prime}$ axis in steady state. In that case, the components of current in the $d^{\prime}$ and $q^{\prime}$ axes can be calculated as:

$\left\{\begin{array}{l}i_{d}^{\prime}=i_{\delta}^{\prime} \sin \theta_{\mathrm{E}}+i_{\gamma}^{\prime} \cos \theta_{\mathrm{E}} \\ i_{q}^{\prime}=i_{\delta}^{\prime} \cos \theta_{\mathrm{E}}-i_{\gamma}^{\prime} \sin \theta_{\mathrm{E}}\end{array}\right.$

As $i_{\gamma}{ }^{\prime}=0$, the torque current $i_{q}{ }^{\prime}$ will have the same sign as $i_{\delta}{ }^{\prime}$ under the restriction of $\theta_{\mathrm{E}} \in(-\pi / 2, \pi / 2)$, that is to say, the LM will also provide braking torque to the MUT if $i_{\delta}{ }^{\prime}<0$. However, according to (11), the current vector $i_{\delta}{ }^{\prime}$ contributes not only to torque current $i_{q}{ }^{\prime}$, but also to the excitation current $i_{d}{ }^{\prime}$. Also, as the angular error trends to zero, the great mass of armature current will act on the $q^{\prime}$ axis. In conclusion, with the restriction of $\theta_{\mathrm{E}} \in(-\pi / 2, \pi / 2)$, the control method above is also available to the case of $\theta_{\mathrm{E}} \neq 0$. In addition, an applied method to reduce $\theta_{\mathrm{E}}$ will be introduced in the experiments section.

\section{Experimental results}

\subsection{Comparative tests for sensorless VC method}

As stated above, the key technology of the proposed efficiency test system is the sensorless VC for the MUT. Once the MUT achieves self-synchronization with $i_{d}=0$, LM can easily supply negative braking torque using two conventional current regulators. Therefore, before the efficiency tests, the experiments were done on a PM servo motor with a photoelectric encoder to verify the effectiveness of the proposed sensorless VC. As with the utilization of the encoder, the actual rotor angle can be

Table 1 Specification of servo motor

\begin{tabular}{ll}
\hline Parameter & Specification \\
\hline Rated output $P_{\mathrm{N}}(\mathrm{kW})$ & 1.5 \\
Rated speed $V_{\mathrm{N}}(\mathrm{r} / \mathrm{min})$ & 2000 \\
Rated torque $T_{\mathrm{N}}(\mathrm{Nm})$ & 5 \\
\hline
\end{tabular}


obtained for comparison. The specification of the servo motor is shown in Table 1.

First, the servo motor was driven by the conventional voltage/frequency (V/F) method to $600 \mathrm{r} / \mathrm{min}$ with no load. During the test, the armature current waveform was captured by the digital oscilloscope and drawn as in Fig. 9a. From the measured data, the RMS of $i_{A}$ is about $2.65 \mathrm{~A}$. Secondly, according to the actual rotor angle, the current components in the $d$ and $q$ axes can be determined from the armature current, and the averages of $i_{d}$ and $i_{q}$ are about 4.21 $\mathrm{A}$ and $0.61 \mathrm{~A}$ respectively as shown in Fig. 9b. It is clear that the controller cannot arrange $i_{d}$ with the $\mathrm{V} / \mathrm{F}$ method. In that case, the great mass of armature current works as a magnetizing component, and the motor works inefficiently.

To compare, the same test was repeated with the proposed sensorless VC. The experimental results are shown in Fig. 10. In contrast to Fig. 9, the RMS of armature current $i_{A}$ decreased from $2.65 \mathrm{~A}$ to about $220 \mathrm{~mA}$, while the current components of $i_{d}$ and $i_{q}$ also declined to around $0 \mathrm{~A}$ and $0.4 \mathrm{~A}$ respectively. As a result, the controller minimizes the magnetizing current, and the motor works in the condition of $i_{d}=0$. In addition, from Fig. 10b, the $\delta$ axis is coincident with the $q$ axis, $\triangle \theta$ converges at zero, and self-synchronization is achieved by the proposed sensorless VC.

Another contrasting experiment was carried out to test the static and dynamic performance of the proposed sensorless VC. The experimental results are shown in Fig. 11. First, Fig. 11a and b shows that the speed and current waveforms in the steady state with two different methods. It is observed that the rotor runs smoothly, and both two sensorless methods can achieve $i_{d}=0$. In conclusion, both the proposed sensorless $\mathrm{VC}$ and the full-order state observer sensorless method [21] have good static performance. However, the proposed control method is simpler than the traditional one. A dynamic test was carried out to verify the dynamic performance by imposing an impact load. From Fig. 11c and d, with the load sudden change, a ripple was sent in both speed and current. In addition, a damped oscillation occurred with the proposed sensorless

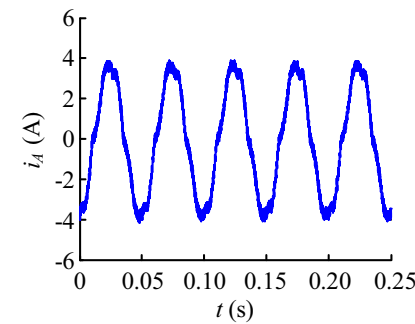

(a) Armature current of servo motor

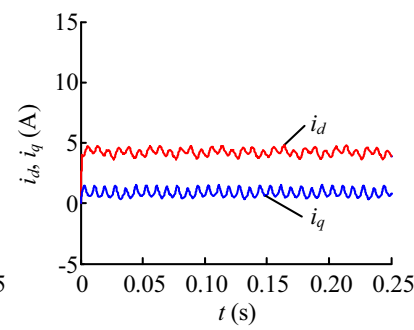

(b) Current components in the $d$ and $q$ axes
Fig. 9 Current waveforms during no-load test in the case of $V / F$ method

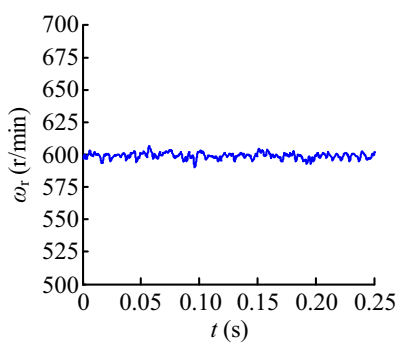

(a) Waveforms of actual rotor speed

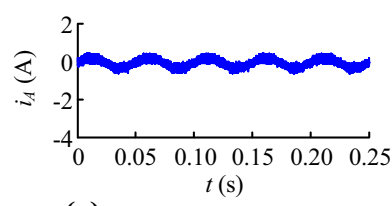

(c) Armature current of servo motor

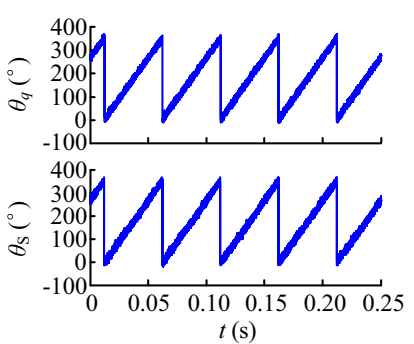

(b) Waveforms of actual rotor angle $\theta_{q}$ \& hypothetical rotor angle $\theta_{\mathrm{S}}$

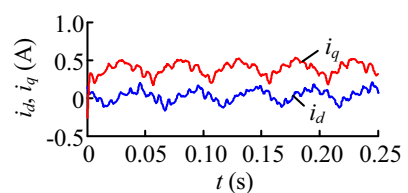

(d) Current components in the $d$ and $q$ axes
Fig. 10 Resulting waveforms during no-load test for VC method

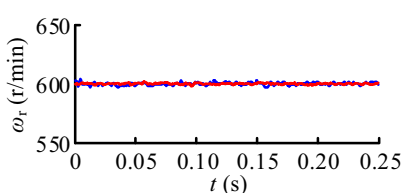

(a) Waveforms of actual rotor speed under steady state

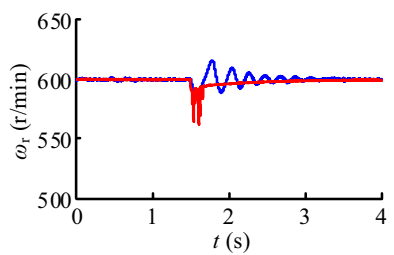

(c) Waveforms of actual rotor speed with sudden load change

— Full-order state observer; - Proposed sensorless VC

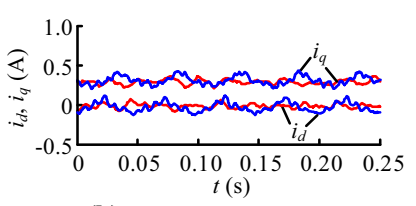

(b) Current components in the $d$ and $q$ axes under steady state

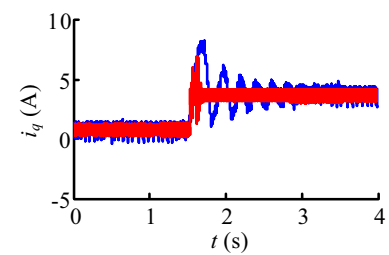

(d) Waveforms of torque current with sudden load change
Fig. 11 Resulting waveforms during the contrast test with two sensorless control methods

VC. As a result, the dynamic performance of the proposed system is inferior to the existing dual closed-loop system.

\subsection{Efficiency tests}

In this section, efficiency tests are carried out for the proposed system. The experiments are done with a digital controller based on TMS320F28335 on a prototype of the proposed system. Two identical high-speed PMSMs are used as the MUT and LM respectively. Two PMSMs are mechanically connected with a high-speed torque meter ET1103 made by Torquemeters Ltd., Britain. A regenerating unit including inverter 1, inverter 2 and common DC bus is used to drive the two PMSMs. A precision power analyzer WT1800 by Yokogawa Ltd. is used to measure the input electrical power of the whole system, the MUT and LM synchronously. In addition, a digital oscilloscope 
WaveRunner $64 \mathrm{Xi}-\mathrm{A}$ is used to measure the armature current of the MUT and LM. The specification of the PMSM is shown in Table 2, and with the use of a magnetic levitation bearing [22], the friction of PMSMs can be ignored.

The block diagram of the efficiency test system is illustrated in Fig. 12. During the experiments, two PMSMs are controlled by one DSP chip to ensure the synchronicity of two hypothetical reference frames: $\delta-\gamma$ frame and $\delta^{\prime}-\gamma^{\prime}$ frame.

As stated in Section 4, there would be an error in the initial rotor position between MUT and LM. Therefore a correction should be made before experimentation. The following processing is performed

1) A static current vector is created in the MUT side to coincide the $d$ axis with the A phase axis to initialize the rotor position of the MUT. This is common in the conventional VC system. As the coupling of two rotors, the rotor of the LM will rotate to a constant position. Then it will record the rotor position of the LM.

2) The same process is performed in the LM side to coincide the $d^{\prime}$ axis with the $\mathrm{A}^{\prime}$ phase axis. The rotor position of LM is again recorded.

3) An angular error can be obtained from the above two positions and a compensation with the angular error should be performed to the initial position of the $\delta^{\prime}-\gamma^{\prime}$ frame. As a result, the initial position difference $\theta_{\mathrm{E}}$ between the MUT and the LM can be approximately eliminated.

The experiment is performed with in three steps:

First, two synchronous hypothetical coordinate systems $\delta-\gamma$ and $\delta^{\prime}-\gamma^{\prime}$ are created on the MUT and the LM side respectively. Considering the inferior performance of the sensorless method at a low speed, a velocity open loop method is used in the MUT side to raise the speed to 3000 $\mathrm{r} / \mathrm{min}$. During this step, there is no need for the braking torque. Therefore, on the LM side, the current commands $i_{\delta}{ }^{\prime}$ and $i_{\gamma}{ }^{\prime}$ are both set to zero to eliminate the armature current. The relative current curves during this step are

Table 2 Specification of high-speed PMSM

\begin{tabular}{ll}
\hline Parameter & Specification \\
\hline Rated output $P_{\mathrm{N}}(\mathrm{kW})$ & 75 \\
Rated voltage $U_{\mathrm{N}}(\mathrm{V})$ & 380 \\
Rated speed $V_{\mathrm{N}}(\mathrm{r} / \mathrm{min})$ & 21000 \\
Rotor inertia $J\left(\mathrm{kgm}^{2}\right)$ & 0.003 \\
Armature resistor $R_{\mathrm{S}}(\mathrm{m} \Omega)$ & 15 \\
Armature induct $L_{\mathrm{S}}(\mu \mathrm{H})$ & 180 \\
\hline
\end{tabular}

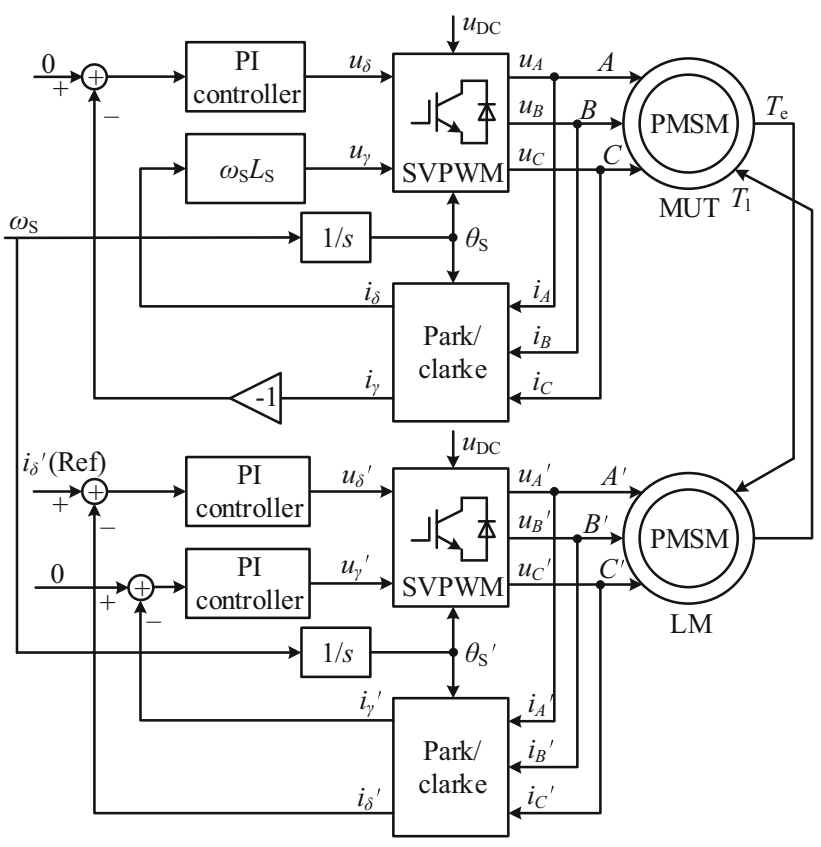

Fig. 12 Block diagram of efficiency test system

drawn in Fig. 13 according to the data captured by the digital oscilloscope WaveRunner 64Xi-A. From Fig. 13a and $b$, in the MUT side, the RMS of the armature current is about $39.6 \mathrm{~A}$, and the current components in the $\delta$ and $\gamma$ axes are $60.88 \mathrm{~A}$ and $-43.86 \mathrm{~A}$ respectively. However, as shown in Fig. $13 \mathrm{c}$ and d, the amplitude in the LM side is much lower with an RMS of $2.5 \mathrm{~A}$ and with the average of $i_{\delta}{ }^{\prime}$ and $i_{\gamma}{ }^{\prime}$ at around zero. According to the torque equation (8), we can find that the MUT works without any load at this step, and the current component mostly acts on the $d$ axis.

Secondly, after the speed reaches $3000 \mathrm{r} / \mathrm{min}$, referring to Fig. 12, the control method for the MUT is switched to the proposed sensorless VC method, while the command for the first PI controller is switched to zero. However, the

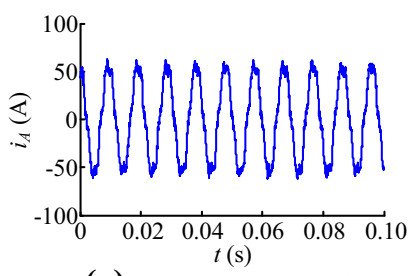

(a) Armature current in the MUT side

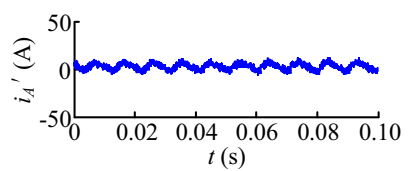

(c) Armature current in the LM side

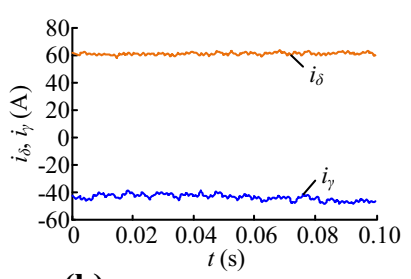

(b) Current components in the $\delta$ and $\gamma$ axes

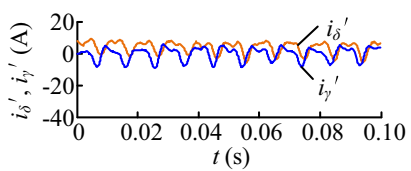

(d) Current components in the $\delta^{\prime}$ and $\gamma^{\prime}$ axes
Fig. 13 Relative current curves during the first step 


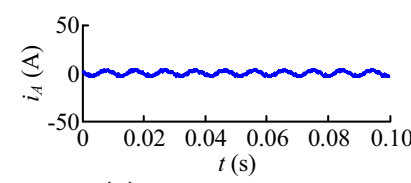

(a) Armature current in MUT side

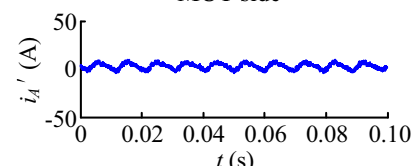

(c) Armature current in LM side

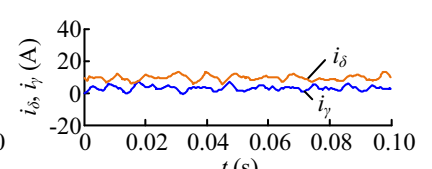

$t(\mathrm{~s})$

(b) Current components in the $\delta$ and $\gamma$ axes

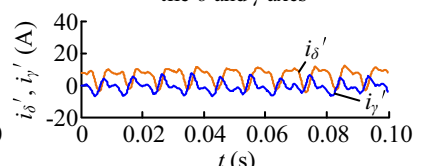

(d) Current components in the $\delta^{\prime}$ and $\gamma^{\prime}$ axes
Fig. 14 Relative current curves during the second step

control method on the LM side is the same as the first step. The resulting curves during this step are shown in Fig. 14. After switching, the armature current of the MUT decreased sharply from $39.6 \mathrm{~A}$ to about $2.3 \mathrm{~A}$, while the current components of $i_{\delta}$ and $i_{\gamma}$ also declined, to $9.68 \mathrm{~A}$ and 0.31 A respectively. However, from Fig. 14c, d, on the LM side, because of the same control method, the armature current and current components are nearly the same as the last step. As a result, using the proposed sensorless VC, the armature current, especially the magnetizing current of the MUT declines much more sharply than the former situation with the same load. In contrast with Fig. 10, we can see that the $\delta$ axis is coincident with the $q$ axis, self-synchronization is achieved, and the MUT works in the condition of $i_{d}=0$.

As stated above, once self-synchronization is achieved on the MUT side, the hypothetical reference frame coincides with the synchronous reference frame on the LM side. This means that after the sensorless VC reaches a steady state, the $\delta^{\prime}$ axis is also coincident with the $q^{\prime}$ axis on the LM side. Then the test turns to the third step. First, the rotation speed of the system is turned up to the rated

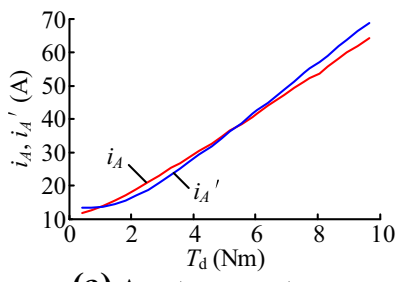

(a) Armature current curves

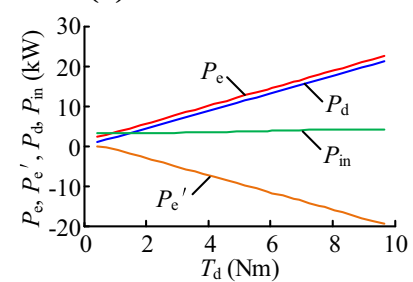

(c) Power curves

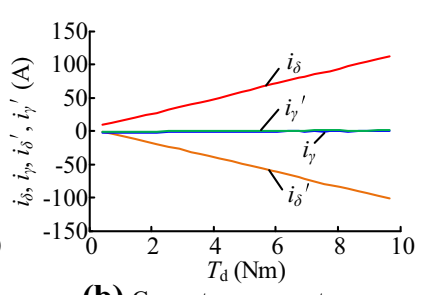

(b) Current component curves

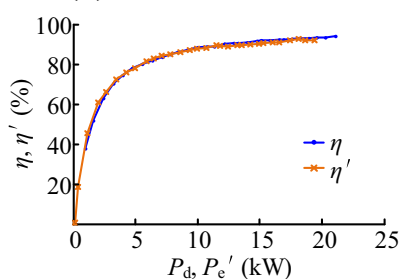

(d) Efficiency curves speed of $21000 \mathrm{r} / \mathrm{min}$ by increasing the reference speed $\omega_{\mathrm{S}}$. Then the torque current $i_{\delta}{ }^{\prime}$ is increased (in the negative direction) by the regulator to load the MUT for the load test. The relative resulting curves are shown in Fig. 15.

Figure 15a shows the responses of armature current at both the MUT and LM sides for the various load torques. It can be seen that the RMS of both $i_{A}$ and $i_{A}{ }^{\prime}$ increase with the increasing load torque with $i_{\mathrm{A}}$ from $11.62 \mathrm{~A}$ to $64.26 \mathrm{~A}$, as well as $i_{A}{ }^{\prime}$ from $13.3 \mathrm{~A}$ to $68.89 \mathrm{~A}$. During the test, the current quality of the LM is inferior to the MUT side with more harmonic components that influence the measured results. Therefore, the RMS of the armature current on the LM side is slightly larger than on the MUT side. The current components on both sides are shown in Fig. 15b, and it is clear that with the (negative) increasing of $i_{\delta}{ }^{\prime}$ (from $-0.98 \mathrm{~A}$ to $-101.19 \mathrm{~A}$ ), more load acts on the MUT. While on the MUT side, because of the sensorless $\mathrm{VC}$, the torque current $i_{\delta}$ automatically climbs from $9.43 \mathrm{~A}$ to $111.85 \mathrm{~A}$ to overcome the load torque. In addition, the magnetizing current $i_{\gamma}$ and $i_{\gamma}{ }^{\prime}$ are around zero all the time. This indicates that for the various torque currents, both the MUT and LM work in the condition of $i_{d}=0$ without any position sensors. The power curves for each part of the system are shown in Fig. 15c. $P_{\mathrm{e}}$ and $P_{\mathrm{e}}^{\prime}$ are the electrical power for the MUT and LM, respectively; $P_{\text {in }}$ is the input power of the whole system from the power grid; and $P_{d}$ is the output mechanical power of the MUT as measured by ET1103. It can be seen that with the increase of load, both $P_{\mathrm{e}}$ and $P_{\mathrm{e}}^{\prime}$ increase obviously as $P_{\mathrm{e}}$ from $2.24 \mathrm{~kW}$ to 22.47 $\mathrm{kW}$ and $P_{\mathrm{e}}{ }^{\prime}$ from $0 \mathrm{~kW}$ to $-19.44 \mathrm{~kW}$. The negative power means the energy circulates from motor to the inverter. However, the total input power of the efficiency test system grows modestly from $3.11 \mathrm{~kW}$ to $4.2 \mathrm{~kW}$ as the losses of two PMSMs and inverters. That is to say, the proposed system achieves the load test with only $4.2 \mathrm{~kW}$ whereas $22.47 \mathrm{~kW}$ is needed for traditional dynamometers. And $81.31 \%$ electrical power is saved by the energy feedback method. The efficiency curves for both MUT and LM are shown in Fig. 15d. With the use of two identical PMSMs, the two efficiency curves are approximately coincident. The final efficiency of the MUT is about $93.95 \%$ with an output power of $21.11 \mathrm{~kW}$.

\section{Conclusion and discussion}

This paper presented a sensorless efficiency test system with energy recovery for a high-speed PMSM. According to the theoretical analysis and experimental verification, the following conclusions can be drawn:

Fig. 15 Results during load test 
1) Using an identical PMSM as the LM, the efficiency test system can achieve the load test without any reduction equipment with a speed of $21000 \mathrm{r} / \mathrm{min}$.

2) During the test, the energy regenerated by the LM can be fed back to the MUT by the regenerating unit, and thereby $81.31 \%$ electrical power is saved.

3) With this sensorless VC method, both the MUT and LM work in the condition of $i_{d}=0$ without a position sensor.

However, the system proposed here still has restrictions:

1) The sensorless VC method is based on estimation of back EMF, thus it is inapplicable at a very low speed.

2) The sensorless VC is independent of the armature resistance $R_{\mathrm{S}}$, but it needs accurate knowledge of the inductance $L_{\mathrm{S}}$.

3) As a single closed-loop control method, the proposed sensorless VC is a little weaker than the existing dual closed-loop system in dynamic performance.

4) The control system of the MUT and LM are interdependent.

Acknowledgements This work was supported by the Science and Technology Project of State Grid Corporation, "Research on Key Technologies of Flexible Control Strategy for Variable Speed Pumped Storage Units" and the Fundamental Research Funds for the Central Universities (No. B18020574).

Open Access This article is distributed under the terms of the Creative Commons Attribution 4.0 International License (http:// creativecommons.org/licenses/by/4.0/), which permits unrestricted use, distribution, and reproduction in any medium, provided you give appropriate credit to the original author(s) and the source, provide a link to the Creative Commons license, and indicate if changes were made.

\section{References}

[1] Sepulchre L, Fadel M,Pietrzak-David M (2016) Improvement of the digital control of a high speed PMSM for vehicle application. In: Proceedings of 11th international conference on ecological vehicles and renewable energies, Monaco, Monaco, 6-8 April 2016, 9 pp

[2] More DS, Fernandes BG (2010) Power density improvement of three phase flux reversal machine with distributed winding. IET Electr Power Appl 4(2):109-120

[3] Yan J, Feng Y, Dong J (2016) Study on dynamic characteristic of wind turbine emulator based on PMSM. Renew Energy 97:731-736

[4] Li L, Zhang J, Zhang C et al (2016) Research on electromagnetic and thermal issue of high-efficiency and high-powerdensity outer-rotor motor. IEEE Trans Appl Supercond 26(4):1-5

[5] Arellano-Padilla J, Asher GM, Sumner M (2006) Control of an AC dynamometer for dynamic emulation of mechanical loads with stiff and flexible shafts. IEEE Trans Ind Electron 53(4):1250-1260
[6] Fodorean D, Viorel IA, Djerdir A et al (2008) Performances for a synchronous machine with optimised efficiency while wide speed domain is attempted. IET Electr Power Appl 2(1):64-70

[7] Balog RS, Sorchini Z, Kimball JW et al (2005) Modern laboratory-based education for power electronics and electric machines. IEEE Trans Power Syst 20(2):538-547

[8] Wang J, Wu M, Jiang P et al (2011) Application of DC electric dynamometer in the PTO power test of tractors. In: Proceedings of international conference on intelligent computation technology and automation, Shenzhen, China, 28-29 March 2011, 4 pp

[9] Yang J, Huang J, Ma J (2005) Research on a novel AC variablefrequency dynamic power dynamometer. In: Proceedings of 8 th international conference on electrical machines and systems, Nanjing, China, 27-29 September 2005, 6 pp

[10] Yang J, Huang J (2006) Research on an AC variable-frequency power dynamometer based on PWM rectifier and fuzzy direct torque control. In: Proceedings of 5th international power electronics and motion control conference, Shanghai, China, 14-16 August 2006, 6 pp

[11] Liu S, Wang M, Li T (2010) Research on direct torque control based asynchronous dynamometer for dynamic emulation of mechanical loads. Kybernetes 39(6):1018-1028

[12] Zhang N, Zhang W (2012) Research on an energy feedback power dynamometer based on improved vector control. In: Proceedings of 15th international conference on electrical machines and systems, Sapporo, Japan, 21-24 October 2012, 4 pp

[13] Chu C, Chou C, Chen J et al (2011) Study of an electric vehicle drive dynamic testing system with energy recovery. In: Proceedings of international conference on power electronics and engineering application, Shenzhen, China, December 2011, 5 pp

[14] Lu K, Lei X, Blaabjerg F (2013) Artificial inductance concept to compensate nonlinear inductance effects in the back EMF-based sensorless control method for PMSM. IEEE Trans Energy Convers 28(3):593-600

[15] Zhu Y, Cheng M, Hua W et al (2013) Sensorless control strategy of electrical variable transmission machines for wind energy conversion systems. IEEE Trans Magn 49(7):3383-3386

[16] Mohammed OA, Khan AA, El-Tallawy AM et al (2012) A wavelet filtering scheme for noise and vibration reduction in highfrequency signal injection-based sensorless control of PMSM at low speed. IEEE Trans Energy Convers 27(2):250-260

[17] Sarikhani A, Mohammed OA (2012) Sensorless control of PM synchronous machines by physics-based EMF observer. IEEE Trans Energy Convers 27(4):1009-1017

[18] Kim H, Son J, Lee J (2011) A high-speed sliding-mode observer for the sensorless speed control of a PMSM. IEEE Trans Ind Electron 58(9):4069-4077

[19] Batzel TD, Lee KY (2005) Electric propulsion with sensorless permanent magnet synchronous motor: implementation and performance. IEEE Trans Energy Convers 20(3):575-583

[20] Liang W, Zhang W, Jiang F (2007) Simulation and comparison of control methods for PMSM servo system. Micromotors 40(1):48-50

[21] Ji L, Xu L (2011) Sensorless control of high speed permanent magnet synchronous motor. Electr Mach Control 15(9):24-30

[22] Ji L, Xu L, Jin C (2013) Research on a low power consumption six-pole heteropolar hybrid magnetic bearing. IEEE Trans Magn 49(8):4918-4926

Li JI received his Ph.D. degree in 2014 from Nanjing University of Aeronautics and Astronautics, Nanjing, China. He now works in the postdoctoral workstation of NARI Group Corporation. His current research interests include the renewable energy generation and energy storage system. 
Yixiang SHAO is currently the vice president of NARI Technology Co., LTD and the manager of electrical control branch. His research interests include smart grid and renewable energy generation.
Sujie GAO is currently the vice president of State Grid Xin Yuan Co., LTD. His research interest includes the technologies of energy storage system. 